\title{
A Novel of Improved algorithm adaptive of NURBS curve
}

\author{
Wan-Jun Zhang ${ }^{1,2,3, a}$, Shan-Ping Gao ${ }^{1, b}$ Su-Jia Zhang ${ }^{1, c}$ \&Feng Zhang ${ }^{2, d}$ \\ 1 Quanzhou Institute of Information Engineering, 36200, China, \\ 2 School of Mechanical Engineering, Xian Jiao tong University, 741049,China \\ 3 Lanzhou Industry and Equipment Co. ,Ltd. , Lanzhou 730050, China
}

Keywords: NURBS curve; improved algorithm adaptive of NURBS; interpolation algorithm; simulation.

\begin{abstract}
In order to solve the problems of a improved algorithm adaptive of NURBS curve,Such as interpolation time bigger, calculation more complicated, and NURBS curve step error are not easy changed and so on. This paper proposed a study on the algorithm for improved algorithm adaptive of NURBS curve and simulation . We can use improved algorithm adaptive of NURBS curve that calculate $\left(x_{i}, y_{i} z_{i}\right)$. Simulation results show that the proposed NURBS curve interpolator meet the high-speed and high-accuracy interpolation requirements of CNC systems. The interpolation of NURBS curve should be finished. The simulation results show that the algorithm is correct; it is consistent with a NURBS curve interpolation requirements.
\end{abstract}

\section{Introduction}

Modern CNC manufacturing systems, NURBS(Non-Uniform Rational B-Spline) has become a mathematical tool used in the field FMS/CIMS. It has many character[1-3]: NURBS can give a unified mathematical representation for surfaces and curves, NURBS can change the shape by modifying weight vector and control point ,etc. But, NURBS has a shortcoming: interpolation time bigger, calculation more complicated, and NURBS curve step error are not easy changed. Lanzhou University of technology and Lanzhou Industry and Equipment Co. Ltd.researchers [4-6] proposed an NURBS algorithms which based on real-time interpolation and adaptive interpolation. Literature [7-9] give some NURBS interpolation algorithms, which makes NC programming complicated and interpolation calculate complicated. Shpitalni et al. [9] derived the same interpolation algorithm by using Taylor's expansion. Houng and Yang [10] were given Cubic spline curve interpolator by using Euler algorithm. Lo and Chung[11-22] proposed the error interpolation algorithm which error calculations changed by curve chord.

On the basis of the research above, a improved algorithm adaptive of NURBS and simulation is presented in this paper. Furthermore, this interpolation algorithm through actual processing of simulation are discussed. The simulation results show that the algorithm is consistent with a NURBS curve interpolation requirements. This interpolation algorithm can meet the high-speed and high-accuracy NURBS curves interpolation requirements.

\section{NURBS Interpolator}

In this paper, NURBS curve is used to represent a parametric of a Improved algorithm adaptive of NURBS curve, and it is introduced first. Supposed $p(u)$ can be represented a Improved algorithm adaptive of NURBS curve. While NURBS [3] are parametrically mathematical definition by the following Eq.(1):

$$
P(u)=\frac{\sum_{i=0}^{n} \omega_{i} d_{i} \mathrm{~N}_{i, k}(u)}{\sum_{i=0}^{n} \omega_{i} \mathrm{~N}_{i, k}(u)}
$$


Where $u$ is cubic time a Improved algorithm adaptive of NURBS curve each parameter, $\mathrm{k}$ the order of a Improved algorithm adaptive of NURBS curve . $p_{i}$ is the control points, $\omega_{i}$ is the weight vector , $N_{i, k}(u)$ is the blending function .

$$
\left\{\begin{array}{c}
N_{i, 0}=\left\{\begin{array}{l}
1 \cdots \cdots \cdots u_{i} \leq u \leq u_{i+1} \\
0 \cdots \cdots \cdots \cdots \cdot \cdots \cdot \text { other }
\end{array}\right. \\
N_{i, k}(u)=\frac{u-u_{i}}{u_{i+k}-u_{i}} N_{i, k-1}(u)+\frac{u_{i+k+1}-u}{u_{i+k+1}-u_{i+1}} N_{i+1, k-1}(u) \\
\text { define } \frac{0}{0}=0
\end{array}\right.
$$

Where the knot vector belong to $U=\left[\begin{array}{lll}u_{i} & , \cdots, & u_{i+k+1}\end{array}\right]$. Based on Eq. (1) and (2), a Improved algorithm adaptive of NURBS curve can be defined when $\omega_{i}, d_{i}, k$ and knot vector are given certain values. a Improved algorithm adaptive of NURBS curve is defined by three types of parameters: Locus of control, Weighted factor and Knot vector. $N_{i, k}$ is shown in Figure1.

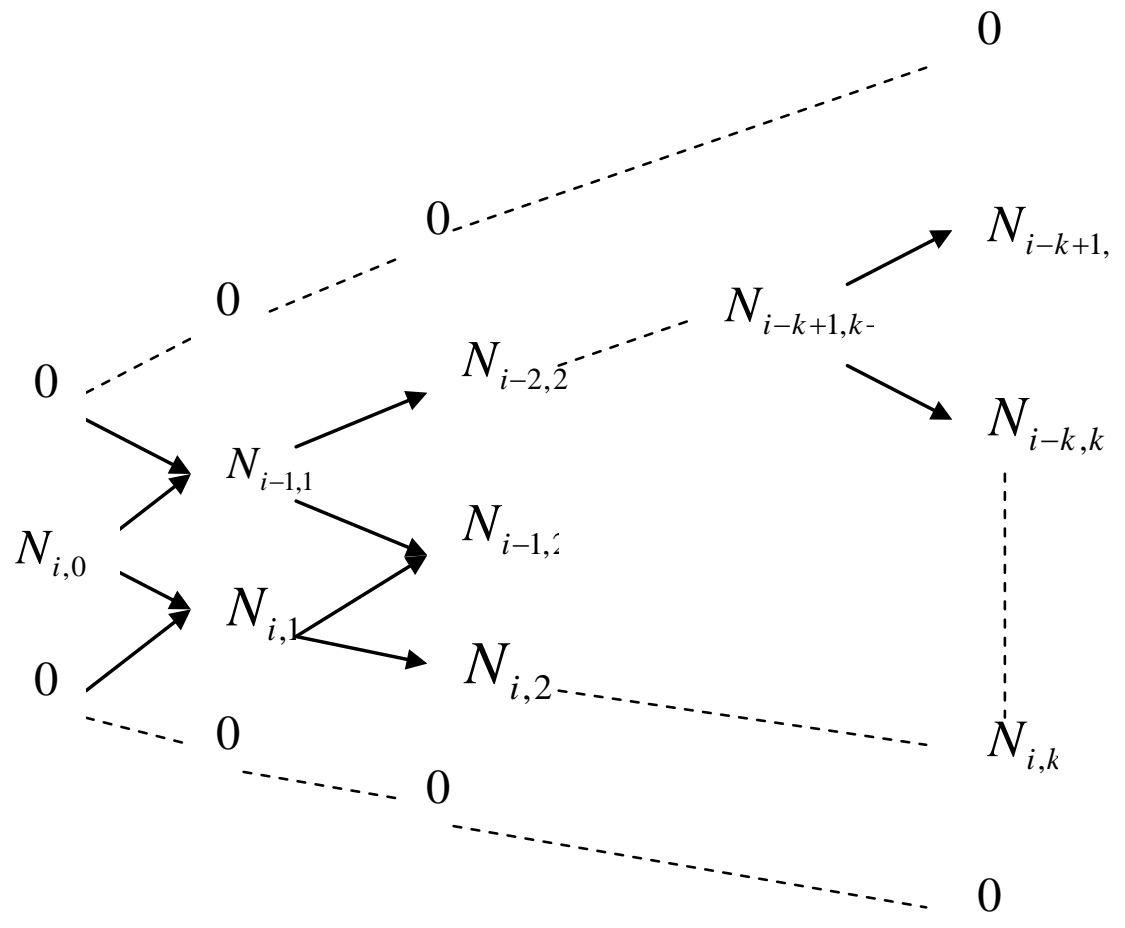

Fig .1: $\quad$ Figure of $N_{i, k}$ formula

Mathematical expression 


$$
\text { Formula: }\left\{\begin{array}{r}
x(u)=\frac{\sum_{i=0}^{n} w_{i} x_{i} N_{i, k}(u)}{\sum_{i=0}^{n} w_{i} N_{i, k}(u)} \\
y(u)=\frac{\sum_{i=0}^{n} w_{i} y_{i} N_{i, k}(u)}{\sum_{i=0}^{n} w_{i} N_{i, k}(u)} \\
z(u)=\frac{\sum_{i=0}^{n} w_{i} z_{i} N_{i, k}(u)}{\sum_{i=0}^{n} w_{i} N_{i, k}(u)}
\end{array}\right.
$$

where $N_{i, k}{ }^{\prime}(u) \mathrm{Ni}, \mathrm{k}(\mathrm{u})$ is a blending function defined by the recursive

$$
N_{i, k}^{\prime}(u)=k\left[\frac{N_{i, k-1}(u)}{u_{i+k}-u_{i}}-\frac{N_{i+1, k-1}(u)}{u_{i+k+1}-u_{i+1}}\right]
$$

Where, $N_{i, k}{ }^{\prime \prime}(u)$ can be represented as follows:

$$
\begin{aligned}
& N_{i, k} "(u)=k(k-1)\left\{\left[\frac{N_{i, k-2}(u)}{\left(u_{i+k}-u_{i}\right)\left(u_{i+k-1}-u_{i}\right)}-\frac{N_{i+1, k-2}(u)}{\left(u_{i+k}-u_{i}\right)\left(u_{i+k}-u_{i+1}\right)}\right]-\right. \\
& \left.\left[\frac{N_{i+1, k-2}(u)}{\left(u_{i+k+1}-u_{i+1}\right)\left(u_{i+k}-u_{i+1}\right)}-\frac{N_{i+2, k-2}(u)}{\left(u_{i+k+1}-u_{i+1}\right)\left(u_{i+k+1}-u_{i+2}\right)}\right]\right\}
\end{aligned}
$$

Supposed $P^{*}(u)=\sum_{i=0}^{n} w_{i} d_{i} N_{i, k}(u), W(u)=\sum_{i=0}^{n} w_{i} N_{i, k}(u)$,

$$
\begin{aligned}
& P(u)=\frac{P^{*}(u)}{W(u)}, \quad \text { we shuold } P^{*}(u) \\
& \text { Supposed } \\
& P^{\bullet}(u)=\frac{d}{d u} P(u)=\frac{1}{W(u)}\left[P^{*^{\bullet}}(u)-W^{\bullet}(u) P(u)\right]
\end{aligned}
$$

where $x(u)$ The derivative of $-u$ is $x^{\prime}(u)$

$$
x^{\prime}(u)=\frac{\sum_{i=0}^{n} w_{i} N_{i, k}(u) \cdot \sum_{i=0}^{n} N_{i, k}{ }^{\prime}(u) w_{i} x_{i}-\sum_{i=0}^{n} w_{i} N_{i, k}{ }^{\prime}(u) \cdot \sum_{i=0}^{n} N_{i, k}(u) w_{i} x_{i}}{\left(\sum_{i=0}^{n} w_{i} N_{i, k}(u)\right)^{2}}
$$

Where $x^{\prime}(u)$ The derivative of- $u$ is $x^{\prime \prime}(u)$

$$
\begin{gathered}
x^{\prime \prime}(u)=\frac{\sum_{i=0}^{n} w_{i} N_{i, k}(u) \cdot \sum_{i=0}^{n} N_{i, k} "(u) w_{i} x_{i}-\sum_{i=0}^{n} w_{i} N_{i, k}{ }^{\prime \prime}(u) \cdot \sum_{i=0}^{n} N_{i, k}(u) w_{i} x_{i}}{\left(\sum_{i=0}^{n} w_{i} N_{i, k}(u)\right)^{2}}- \\
\frac{2 \sum_{i=0}^{n} w_{i} N_{i, k}{ }^{\prime}(u)\left(\sum_{i=0}^{n} w_{i} N_{i, k}(u) \cdot \sum_{i=0}^{n} N_{i, k}{ }^{\prime}(u) w_{i} x_{i}-\sum_{i=0}^{n} N_{i, k}(u) w_{i} x_{i} \cdot \sum_{i=0}^{n} w_{i} N_{i, k}(u)\right)}{\left(\sum_{i=0}^{n} w_{i} N_{i, k}(u)\right)^{3}}
\end{gathered}
$$


Where $y(u)$ The derivative of- $u$ is $y^{\prime}(u)$

$$
y^{\prime}(u)=\frac{\sum_{i=0}^{n} w_{i} N_{i, k}(u) \cdot \sum_{i=0}^{n} N_{i, k}{ }^{\prime}(u) w_{i} y_{i}-\sum_{i=0}^{n} w_{i} N_{i, k}(u) \cdot \sum_{i=0}^{n} N_{i, k}(u) w_{i} y_{i}}{\left(\sum_{i=0}^{n} w_{i} N_{i, k}(u)\right)^{2}}
$$

Where $y^{\prime}(u)$ The derivative of- $u$ is $y^{\prime \prime}(u)$

$$
\begin{gathered}
y^{\prime \prime}(u)=\frac{\sum_{i=0}^{n} w_{i} N_{i, k}(u) \cdot \sum_{i=0}^{n} N_{i, k}{ }^{\prime \prime}(u) w_{i} y_{i}-\sum_{i=0}^{n} w_{i} N_{i, k}{ }^{\prime \prime}(u) \cdot \sum_{i=0}^{n} N_{i, k}(u) w_{i} y_{i}}{\left(\sum_{i=0}^{n} w_{i} N_{i, k}(u)\right)^{2}}- \\
\frac{2 \sum_{i=0}^{n} w_{i} N_{i, k}(u)\left(\sum_{i=0}^{n} w_{i} N_{i, k}(u) \cdot \sum_{i=0}^{n} N_{i, k}{ }^{\prime}(u) w_{i} y_{i}-\sum_{i=0}^{n} N_{i, k}(u) w_{i} y_{i} \cdot \sum_{i=0}^{n} w_{i} N_{i, k}{ }^{\prime}(u)\right)}{\left(\sum_{i=0}^{n} w_{i} N_{i, k}(u)\right)^{3}}
\end{gathered}
$$

Where $z(u)$ The derivative of $-u$ is $z^{\prime}(u)$

$$
z^{\prime}(u)=\frac{\sum_{i=0}^{n} w_{i} N_{i, k}(u) \cdot \sum_{i=0}^{n} N_{i, k}{ }^{\prime}(u) w_{i} z_{i}-\sum_{i=0}^{n} w_{i} N_{i, k}{ }^{\prime}(u) \cdot \sum_{i=0}^{n} N_{i, k}(u) w_{i} z_{i}}{\left(\sum_{i=0}^{n} w_{i} N_{i, k}(u)\right)^{2}}
$$

Where $z^{\prime}(u)$ The derivative of- $u$ is $z^{\prime \prime}(u)$

$$
\begin{aligned}
& z^{\prime \prime}(u)=\frac{\sum_{i=0}^{n} w_{i} N_{i, k}(u) \cdot \sum_{i=0}^{n} N_{i, k}{ }^{\prime \prime}(u) w_{i} z_{i}-\sum_{i=0}^{n} w_{i} N_{i, k}{ }^{\prime \prime}(u) \cdot \sum_{i=0}^{n} N_{i, k}(u) w_{i} z_{i}}{\left(\sum_{i=0}^{n} w_{i} N_{i, k}(u)\right)^{2}}- \\
& \frac{2 \sum_{i=0}^{n} w_{i} N_{i, k}{ }^{\prime}(u)\left(\sum_{i=0}^{n} w_{i} N_{i, k}(u) \cdot \sum_{i=0}^{n} N_{i, k}{ }^{\prime}(u) w_{i} z_{i}-\sum_{i=0}^{n} N_{i, k}(u) w_{i} z_{i} \cdot \sum_{i=0}^{n} w_{i} N_{i, k}(u)\right)}{\left(\sum_{i=0}^{n} w_{i} N_{i, k}(u)\right)^{3}}
\end{aligned}
$$

The Taylor expansion of parameter $\mathrm{u}$ to time $\mathrm{t}$, the corresponding approximated algorithm can be obtained.

$$
u_{i+1}=u_{i}+\left.\frac{d u}{d t}\right|_{t=t_{i}} T+\left.\frac{1}{2} \frac{d^{2} u}{d t^{2}}\right|_{t=t_{i}} T^{2}+\text { H.O.T. }
$$

The second-order expansion of Taylor formula

$$
u_{i+1}=u_{i}+\frac{V T}{\sqrt{\left(x^{\prime}\right)^{2}+\left(y^{\prime}\right)^{2}+\left(z^{\prime}\right)^{2}}}+\frac{(V T)^{2} \times\left(x^{\prime} x^{\prime \prime}+y^{\prime} y^{\prime \prime}+z^{\prime} z^{\prime \prime}\right)}{2\left(\left(x^{\prime}\right)^{2}+\left(y^{\prime}\right)^{2}+\left(z^{\prime}\right)^{2}\right)^{2}}
$$

From Eq.(10), Eq.(11), Eq.(12), Eq.(13),Eq.(14), Eq.(15) we should get $\left(x_{i}, y_{i}, z_{i}\right)$, a Improved algorithm adaptive of NURBS curve should be finished.

\section{A Improved algorithm of flow chart adaptive of NURBS curve}

Figure 2 for Flowchart of algorithm. a Improved algorithm adaptive of NURBS curve is explained as follow: 
Step1: Input NURBS curve paramter, such as NURBS curve control points, weight vector and so on.

Step2: Calculate Taylor's expansion.

Step3: Calculate $x^{\prime}, x^{\prime \prime}, y, y^{\prime \prime}, z^{\prime}, z^{\prime \prime}$.

Step4:Get NURBS curve position ${ }^{\left(X_{i}, y_{i}, Z_{i}\right)}$ can be calculated by using m Improved algorithm adaptive of NURBS curve.

Step5: NURBS curve interpolation is finished.

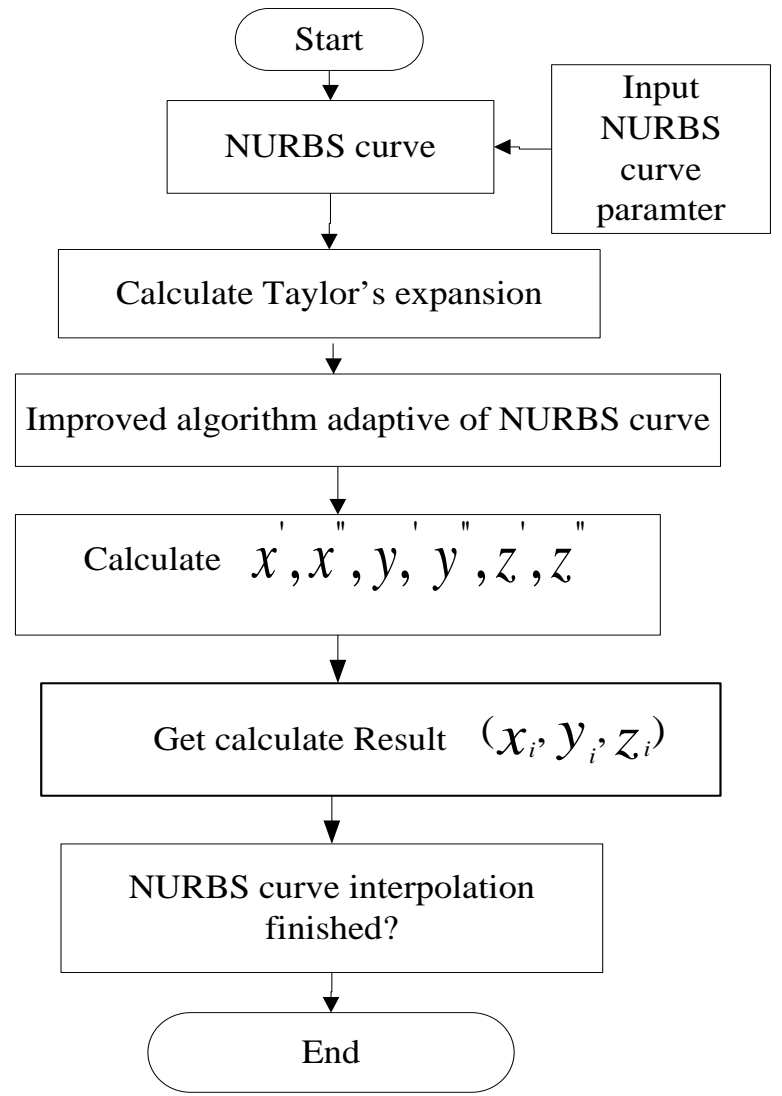

Fig .2: Flowchart of algorithm

\section{Experiment simulation and data analysis}

In this simulation ,this interpolation scheme is realized on the motion controller developed by our own lab, based on DSP TMS20C543,Development environment is a PC with AMD Sempron $2,800+2.1 \mathrm{Ghz}$ CPU,2GB RAM, and main frequency is $1.44 \mathrm{MHz}$,machine tool is machine center. Machining parameters and dynamics parameters are shown in Table 1.

In the paper, the interpolation of improved algorithm adaptive of NURBS is utilized as an example to the Newton-Rapson iterative algorithm. The control points, weight vector, and knot vector of NURBS for the provided example are assigned as follows:

The control pointsare $P=\left[\begin{array}{l}0 \\ 0 \\ 0\end{array}\right]\left[\begin{array}{l}1 \\ 1 \\ 2\end{array}\right]\left[\begin{array}{l}2 \\ 1 \\ 2\end{array}\right]\left[\begin{array}{c}3 \\ 1.5 \\ 2.5\end{array}\right]\left[\begin{array}{c}3 \\ 2 \\ 2.5\end{array}\right]\left[\begin{array}{c}3 \\ 3 \\ 3.5\end{array}\right]\left[\begin{array}{c}3 \\ 1.15 \\ 3.5\end{array}\right]$ (unit: $\mathrm{mm}$ ),

The weight vector is $\omega_{i}=\left\{\begin{array}{llllll}0 & 0 & 0.405 & 0.0636 & 0.742 & 0.742\end{array}\right\}$,

and the knot vector is $u=\left[\begin{array}{lllllllll}1.6 & 1.7 & 0.7 & 1.25 & 0.6 & 0.85 & 1 & 1.2 & 1.25\end{array}\right]$. 
Tab. 1 Machining parameters and dynamics parameters

\begin{tabular}{ccccccc}
\hline$J\left(\mathrm{~mm} \cdot \mathrm{s}^{-2}\right)$ & $F\left(\mathrm{~mm} \cdot \mathrm{s}^{-1}\right)$ & $T(\mathrm{~ms})$ & $\delta_{\text {max }}(\mu \mathrm{m})$ & $V_{\text {x max }}\left(\mathrm{mm} \cdot \mathrm{s}^{-1}\right)$ & $V_{\text {ymax }}\left(\mathrm{mm} \cdot \mathrm{s}^{-1}\right)$ & $V_{z \max }\left(\mathrm{mm} \cdot \mathrm{s}^{-1}\right)$ \\
\hline 38100 & 240 & 1.15 & 1.015 & 60 & 85 & 55 \\
\hline
\end{tabular}

$J$ the allowable acceleration and jerk, $F$ command interpolation federate, $T$ the interpolation period, $\delta_{\max }$ the maximum value of the chord error, $v_{x \max }$ the max feed rate vale of x-axis, $V_{y \max }$, the max feed rate vale of y-axis, $V_{z \max }$ the max feed rate vale of z-axis. By Using computer soft in NURBS curve interpolation, as shown in Tab.2, Figure 3,Figure 4 and Figure 5.

$G$ code

G01 X16.549000 Y0.010000 Z-14.222000

G01 X16.447000 Y0.739000 Z-14.096000

G01 X16.349000 Y1.375000 Z-13.986000

G01 X16.170000 Y2.386000 Z-13.829000

G01 X16.003000 Y3.213000 Z-13.728000

G01 X15.892000 Y3.714000 Z-13.678000

G01 X13.913000 Y12.634000 Z-15.078000

G01 X13.882000 Y13.136000 Z-15.309000

G01 X13.876000 Y13.444000 Z-15.446000

G01 X13.869000 Y13.560000 Z-15.502000

G01 X13.862000 Y13.703000 Z-15.564000

G01 X13.855000 Y13.846000 Z-15.626000

NURBS Code $\downarrow$

NURBS p4 \{0.000000, 0.000000, 0.000000, 0.000000,

$0.385549\}$ X16.549000 Y0.010000 Z- 14.222000 w1.000000

k0.502421 X16.323334 Y1.827069 Z-13.890654 w1.000000

k0.606291 X15.814100 Y4.190536 Z-13.515593 w1.000000

k0.711405 X15.082927 Y7.029935 Z-13.619467 w1.000000

k0.810409 X14.692049 Y8.546189 Z-13.792276 w1.000000

k1.000000 X14.349083 Y9.960615 Z-14.103859 w1.000000

k1.000000 X13.975937 Y11.749510 Z-14.652294 w1.000000

k1.000000 X13.871343 Y13.008857 Z-15.255747 w1.000000

The FANUC CNC system has a large share in the current CNC system market, and they make these parameters as part of the NC program command parameters, Interpolation G code shown in Figure 1:

G06.2 K3 U0 X0 Y0 Z1 W1.6 F18

U0 X25 Y30 Z6 W1.7

U0 X50 Y50 Z16 W0.7

U0 X65 Y60 Z22 W1.25

U0.4531 X77 Y70 Z30 W0.6

U0.5485 X105 Y57 Z32 W0.85

U0.6306 X132 Y40 Z41 W1

U0.7426 X142 Y30 Z51 W1.2

U1 X152 Y10 Z21 W1.2

$\mathrm{U} 1$

U1 


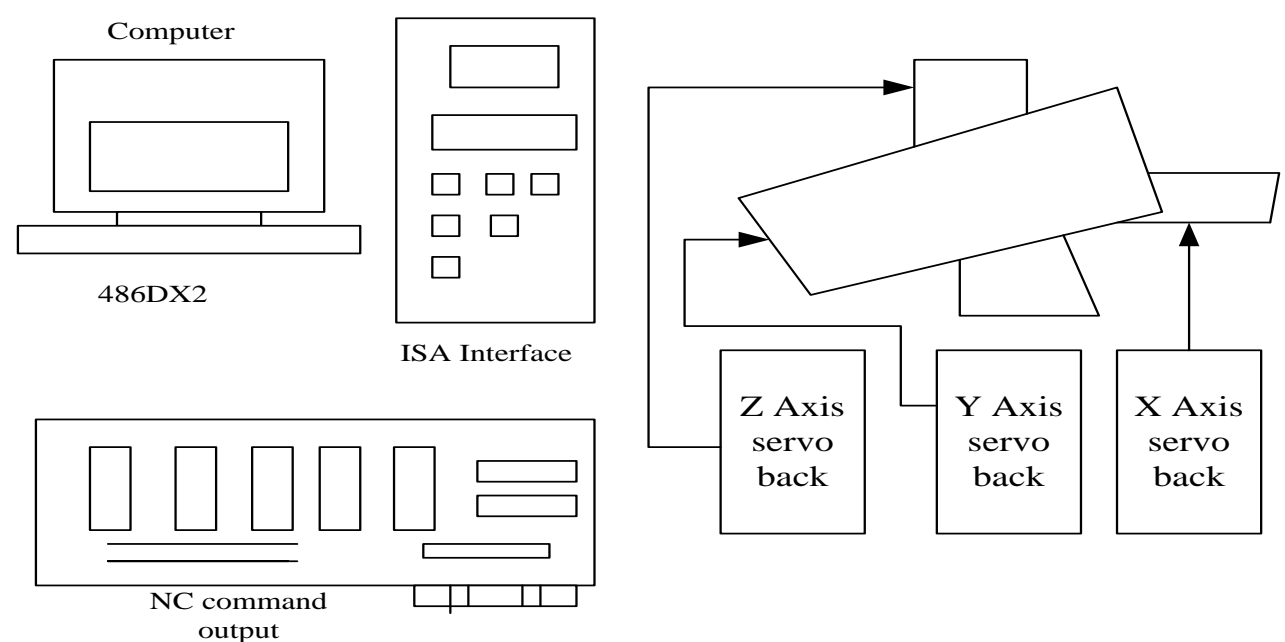

Fig .3: The experimental setup

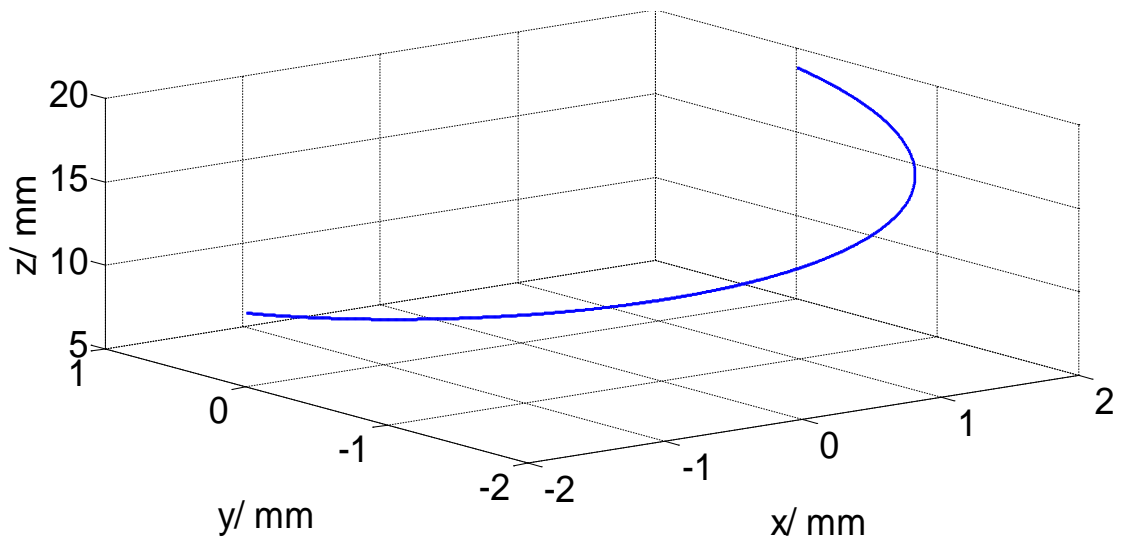

Fig. 4 : NURBS curve interpolation

Table 2 Partial interpolation results of NURBS curve

\begin{tabular}{clllll}
\hline Number of steps & $\mathrm{u}$ & $\mathrm{X}(\mathrm{mm})$ & $\mathrm{Y}(\mathrm{mm})$ & $\mathrm{Z}(\mathrm{m})$ & $\triangle \mathrm{L}(\mathrm{mm})$ \\
\hline 420 & 0.51 & 0.634955 & 0.90286 & 18.4648 & 0.297 \\
421 & 0.514 & 0.637206 & 0.59191 & 18.5699 & 0.3 \\
422 & 0.5181 & 0.639471 & 0.593553 & 18.6782 & 0.3 \\
423 & 0.5223 & 0.641723 & 0.595198 & 18.7886 & 0.3 \\
424 & 0.5264 & 0.643961 & 0.596847 & 18.9012 & 0.3 \\
425 & 0.5306 & 0.646186 & 0.5985 & 18.0159 & 0.2665 \\
426 & 0.5342 & 0.638152 & 0.599974 & 18.1198 & 0.2387 \\
427 & 0.5373 & 0.649895 & 0.601291 & 18.3139 & 0.2192 \\
428 & 0.54 .2 & 0.651494 & 0.602507 & 19.3017 & 0.2062 \\
429 & 0.5428 & 0.65229 & 0.603653 & 19.3853 & 0.1967 \\
430 & 0.5452 & 0.654412 & 0.604749 & 19.4657 & 0.1894 \\
431 & 0.5475 & 0.655775 & 0.605805 & 20.5439 & 0.1845 \\
432 & 0.5497 & 0.657097 & 0.606836 & 20.6207 & 0.1851 \\
433 & 0.5518 & 0.658421 & 0.607872 & 20.6983 & 0.1861 \\
434 & 0.5539 & 0.659749 & 0.608912 & 21.7767 & 0.1872 \\
435 & 0.556 & 0.661084 & 0.609956 & 21.856 & 0.1883 \\
\hline
\end{tabular}




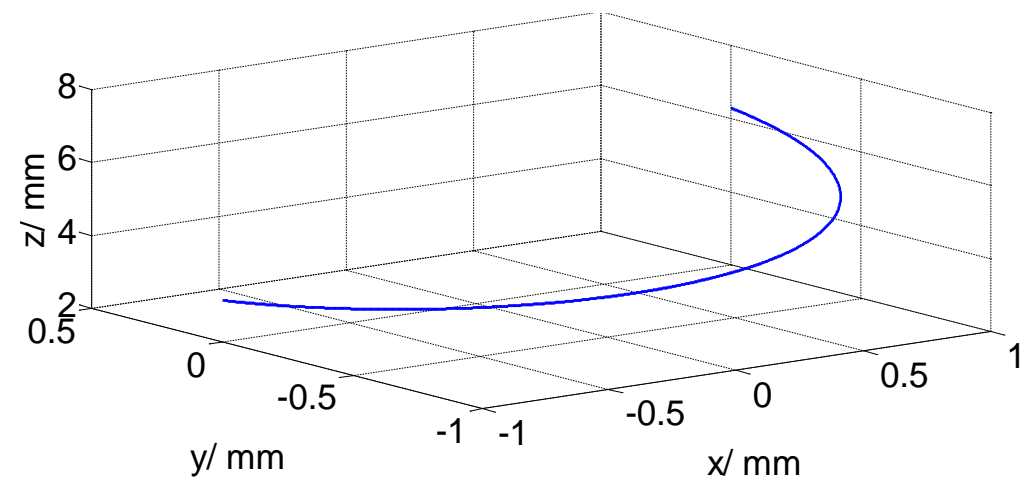

Fig. 5 : Improved algorithm adaptive of NURBS

Table 3 Partial interpolation results of Improved algorithm adaptive of NURBS

\begin{tabular}{|c|c|c|c|c|c|}
\hline Number of steps & $\mathrm{u}$ & $\mathrm{X}(\mathrm{mm})$ & $\mathrm{Y}(\mathrm{mm})$ & $\mathrm{Z}(\mathrm{m})$ & $\triangle \mathrm{L}(\mathrm{mm})$ \\
\hline 420 & 0.515 & 0.6349558 & 0.5902868 & 6.4648 & 0.2975 \\
\hline 421 & 0.5145 & 0.6372068 & 0.5919177 & 6.5699 & 0.355 \\
\hline 422 & 0.51815 & 0.6394718 & 0.593553 & 6.6782 & 0.366 \\
\hline 423 & 0.52235 & 0.6417236 & 0.5951986 & 6.7886 & 0.322 \\
\hline 424 & 0.52645 & 0.6439617 & 0.5968475 & 6.9012 & 0.3224 \\
\hline 425 & 0.53065 & 0.6461864 & 0.598544 & 6.0159 & 0.26651 \\
\hline 426 & 0.53425 & 0.6381525 & 0.5999744 & 6.1198 & 0.23871 \\
\hline 427 & 0.53735 & 0.6498952 & 0.6012913 & 6.3139 & 0.21922 \\
\hline 428 & 0.54 .25 & 0.6514943 & 0.6025071 & 6.3017 & 0.20623 \\
\hline 429 & 0.54285 & 0.652291 & 0.6036532 & 6.3853 & 0.19674 \\
\hline 430 & 0.54525 & 0.65441222 & 0.6047494 & 6.4657 & 0.18944 \\
\hline 431 & 0.54755 & 0.65577533 & 0.6058053 & 6.5439 & 0.18455 \\
\hline 432 & 0.54975 & 0.6570973 & 0.6068365 & 6.6207 & 0.18513 \\
\hline 433 & 0.55185 & 0.6584214 & 0.6078724 & 6.6983 & 0.18611 \\
\hline 434 & 0.55396 & 0.6597495 & 0.6089128 & 6.7767 & 0.18721 \\
\hline 435 & 0.5566 & 0.6610846 & 0.6099567 & 6.856 & 0.18831 \\
\hline
\end{tabular}

Tab.4 Table analysis of interpolation of NURBS curve results

\begin{tabular}{lccc}
\hline \multicolumn{1}{c}{ Parameters algorithms } & Interpolation time(s) & $\begin{array}{c}\text { Max step } \\
\text { error (mm) }\end{array}$ & $\begin{array}{c}\text { Min step } \\
\text { error (mm) }\end{array}$ \\
\hline NURBS interpolation & 17.84 & 9.0801 & 0.712 \\
Newton-Rapson iterative interpolation & 15.5 & 7.9801 & 0.46 \\
\hline
\end{tabular}

As can be seen from Fig. 4, Fig.5 and table 1 and table 2 , in the process of the interpolation, interpolation time reduced, Max step error deceased, Max step error value is 7.980, which meet the expected to interpolation, i.e. to reduce the compensation error and interpolation step chord error. To verify the high efficiency and reliability of this improved algorithms adaptive of NURBS are applied in the experiments to make a comparison. It can be seen that mproved algorithm adaptive of NURBS is feasible and efficent.

In a word, the algorithm for improved algorithm adaptive of NURBS presented in this paper could satisfy high-speed and high-accuracy interpolation requirements, which can be used for actual interpolation processing.

\section{Conclusions}

In the paper,Study on improved algorithm adaptive of NURBS and simulation is introduced. We can use Improved algorithm adaptive of NURBS that calculate $\left(x_{i}, y_{i}, z_{i}\right)$. Simulation results show that the proposed NURBS curve interpolator meet the high-speed and high-accuracy interpolation requirements of CNC systems. The interpolation of NURBS curve should be finished. Simulation results show that the proposed NURBS curve interpolator meet the high-speed and high-accuracy interpolation requirements of CNC systems, it is consistent with a NURBS curve interpolation requirements. In addition ,NC machining time can be reduced. Implementation on NC machine has proven the feasibility of a developed interpolation algorithm. 


\section{Acknowledgements}

The authors thank the financial supports from National Natural Science Foundation of China(Grant no. 51165024) and Science and Technology Major Project of "High-grade NC Machine Tools and Basic Manufacturing Equipment” (2010ZX040001-181).

\section{References}

[1] Fang Yi-Xiang, Liu Wen-Xue. Based on the geometric properties of the cubic uniform B-Spline curve structure description[J]. Journal of engineering graphics, 2 , pp.96-102, 2006.

[2] Zhang Wan-Jun, Hu Chi-Bing, Zhang Feng, et al . Honing machine motion control card three B spline curve method of interpolation arithmetic for CNC system [J]. Chinese Journal of Manufacturing Technology \& Machine Tool , 8(8), pp.40-43,August 2012.

[3] Zhang Wan-Jun, HU Chi-bing, WU Zai-xin, et,al. Research on modification algorithm of Three B Spline curve interpolation technology [J]. Chinese Journal of Manufacturing Technology \& Machine Tool , 2 pp.141-143,Feburary 2013.

[4] Zhang Wanjun, Zhang Feng, Zhang Guohua. Research on a algorithm of adaptive interpolation for NURBS curve. [J].Applied Mechanics and Materials , Vol. 687-691, pp.1600-1603, December 2014.

[5] Zhang Wan-Jun, Zhang Feng, Zhang Guohua. Research on modification algorithm of Cubic Bspline curve interpolation technology. [J].Applied Mechanics and Materials, Vol. 687-691, pp.1596-1599, December 2014.

[6] Zhang Wan-Jun, Zhang Feng, Zhang Wan-Liang. Research on a NURBS curve of timing / interrupt interpolation algorithm for CNC system [J]. Chinese Journal of Manufacturing Technology \& Machine Tool , 4(4), pp.40-43,April 2015.

[7] Kong Fan-Guo, Hao Shang-Hua, ZhongYan-Zhi. NURBS curve interpolation algorithm to achieve the VC [J]. China new technology and new products, 17, pp.8-9, 2009.

[8] Ye Bo-Sheng,Yang Shu-Zi. CNC system in cubic B-Spline curve interpolation method [J ]. China Mechanical Engineering, 9 ( 3) , pp. 42 - 43, 1998.

[9] Li He-Cheng, Wang Yu-Ping, An interpolation based genetic algorithm for sloving nonlinear bilevel programming problems.Chinese Journal of Computers, 31(6), pp.910-918, June 2008.

[10] Shpitalni M, Koren Y, Lo CC. Realtime curve interpolators. Computer- Aided Design, 26, pp.832-838, 1994.

[11] Huang JT, Yang DCH. A generalized interpolator for command generation of parametric curves in computer controlled machines. Japan/USA Symposium on Flexible Automation,1(1), pp.393-399, January1992.

[12] Lo CC, Chung CY. Curve generation and control for biaxial machine tools. J. CSME , 18, pp.175-182, 1997.

[13]Lo CC,Chung CY.Curve generation and control for biaxial machine tools. J.CSME ,18, pp.175-182,1997.

LI Haiyang, ZHAO Yugang, HU Liu. The Improvement Study on Involute Profile Type Rotor Profile in Roots Vacuum Pump [J]. MACHINE TOOL\&HYDRAULICS , 2011,39(22): 37-39.

[14]Hang Houjun, Chou Ru. Investigation of practical representation and parameterization of the rational

cubic conic sections. TELKOMNIKA Indonesian Journal of Electrical Engineering. 2013; 11(1): 918.

[15] Zhang wanjun.A Newton iteration-Rapson of NC interpolation system [P]. China : 201520043082.8,2015.

[16] Zhang wanjun. Based on b-spline curve real-time interpolation of CNC system at a high speed [P]. China : 201520069002.6,2015. 
[17] Zhang wanjun. A kind of based on ARM + FPGA dual CPU of NC interpolation system [P]. China : 201520969163. 0,2016.

[18] Zhang wanjun. A control system of numerical control for interpolation PCI system [P]. China : 201620110057.1,2016.

[19] Sun, Shujie, Lin, Hu; Zheng, Liaomo. A real-time and look-ahead interpolation methodology with dynamic B-spline transition scheme for CNC machining of short line segments [J ]. Internation journal of advanced manufacturing technology, 84 ( 3) , pp. 1359-1370, 2016.

[20] Duan, Molong, Okwudire, Chinedum. Minimum-time cornering for CNC machines using an optimal control method with NURBSparameterization

[J]. Internation journal of advanced manufacturing technology, 85 ( 3) , pp. 1405-1418, 2016.

[21]Liu Qiang,Liu Huan,Yuan Songmei.High Accurate Interpolation of NURBS Tool Path for CNC Machine Tools[J]. China journal of mechanical engineering, 29 ( 5) , pp. 911-920, 2016.

[22] Zhang Liqiang,Zhang, Kai Yan, Yecui.Local Corner Smoothing Transition Algorithm Based on Double Cubic NURBS for Five-axis Linear Tool Path[J].Strojniskivestnik-journal of mechanical engineering, 29 ( 5) , pp. 647-656 , 2016(9). 